\title{
Beyond ROI: Using Value of Investment to Measure Employee Health and Wellness
}

\author{
Ronald J. Ozminkowski, PhD, Seth Serxner, $\mathrm{PhD}^{2}$, Karen Marlo, MPP, \\ Rohit Kichlu, MBA, Erin Ratelis, MBC, and Jennifer Van de Meulebroecke ${ }^{6}$
}

\section{Introduction}

$\mathbf{F}$ OR YEARS, EMPLOYERS HAVE VIEWED return on investment (ROI) estimates derived from health care cost savings as the preferred method to assess the impact of employee health and wellness programs. ${ }^{1}$

However, over the last few years, a movement toward a broader business case for health management is gaining momentum. The decades-old method of using only a financial metric (health care cost savings) to measure the value of health and wellness programs is on the wane. ${ }^{2}$ Gradually rising in its place is a value-of-investment (VOI) approach. ${ }^{3}$

VOI incorporates many other metrics to estimate program impact. Examples include employee morale, reduced turnover, business profitability, health risk reduction, reduced sick or disability days, higher productivity at work, and increased quality of life. ${ }^{4,5}$

To test the notion of VOI primarily with human resource professionals, Optum, the National Business Group on Health, and TRC Market Research conducted a survey of 275 employers. The goal of the survey was to determine if employers' reasons for investing in health promotion goes beyond medical expenditure savings. If so, it may be time for the industry to more fully engage in a VOI-based discussion when considering such investments and when estimating the total value obtained from health and wellness programs.

\section{The Survey}

The survey sample included 275 respondents from employers who offered at least 5 different health and wellness programs. These programs could include case management to address the needs of patients with many comorbid health conditions, various types of disease management programs for patients with 1 or only a few chronic conditions, and wellness programs such as weight management programs, physical activity programs, and tobacco cessation programs.

Survey respondents were involved in health benefits decision making and nearly $90 \%$ were at the director level or above within their company. More than $90 \%$ of the survey respondents had 3000 or more employees. Sample employers represented a wide range of industries including manufacturing, health care, retail, professional/technical, and financial/insurance.

The research study was designed to answer a critical question: Are employers offering health and wellness programs for reasons beyond health care cost savings? Taking that premise a step further, the research study sought to identify the:

- Primary and emerging reasons employers offer health and wellness programs

- Relative importance of each reason

- Metrics currently used to demonstrate program value, and

- Ease with which employers can measure and track outcomes.

Survey respondents were interviewed between August and September 2014. All 275 respondents participate in an ongoing survey panel constructed and maintained by Research Now Group, Inc., contracted by TRC Market Research. Panel respondents were compensated by Research Now for their participation. Results from the survey have a $+/-5.9 \%$ margin of error.

\section{Analysis}

Maximum difference scaling (MaxDiff) was used to determine the relative importance of the reasons identified by survey respondents for investing in health and wellness programs. MaxDiff is a statistical technique used to understand the relative importance among a list of items. ${ }^{6}$ The MaxDiff

\footnotetext{
${ }^{1}$ Consumer Solutions Group, Optum, Ann Arbor, Michigan.

${ }^{2}$ Consumer Solutions Group, Optum, Minneapolis, Minnesota.

${ }^{3}$ National Business Group on Health, Washington, DC

${ }^{4}$ Optum, East Windsor, New Jersey.

${ }^{5}$ Optum, Milwaukee, Wisconsin.

${ }^{6}$ TRC Market Research, Fort Washington, Pennsylvania.
} 
approach provides respondents with sets of 3 items and asks them to choose the most important and least important one within each set. These items are then selected to appear in many subsequent item sets in order to better identify respondent priorities.

As the survey progresses the MaxDiff analysis process incorporates hierarchical Bayesian analyses to estimate the relative preference of each reason for investing in health and wellness programs. Bayesian techniques are useful because they assess relative preference by incorporating knowledge expressed by survey respondents in previous item sets within the survey. Relative preferences were transformed to scores on a $0-100$ scale. These scores show the importance of reasons compared to each other. For example, a score of 20 can be interpreted as twice as important as a score of 10 .

\section{Findings}

There were 4 key insights from the survey.

Key insight \#1: Employers are already focused on building a broader business case for health and wellness programs.

Approximately $91 \%$ of respondents reported offering health and wellness programs for reasons other than medical cost savings.

More specifically, the study found that the top 3 reasons employers offer health and wellness programs are to:

- Reduce employee health risks (MaxDiff score =13.44)

- Reduce health care costs (MaxDiff score=13.35).

- Improve employee productivity (MaxDiff score $=9.45$ ).

As can be seen from the MaxDiff scores, these 3 reasons were not of equal weight. The MaxDiff method allowed us to understand the relative importance of each reason. For example, we found that health risk reduction and health care cost savings were more than $40 \%$ more important to employers than were productivity improvements (eg, the MaxDiff score of 13.44 for reducing health risks is 1.42 times as high [or approximately $42 \%$ higher], relatively speaking, than the score of 9.45 for improving productivity).

Key insight \#2: In addition to the top 3 reasons mentioned, a cluster of 8 emerging reasons are driving employers to offer health and wellness programs.

Although the top 3 reasons were not surprising, the research revealed a cluster of emerging reasons that represent the VOI opportunity. Along with their MaxDiff scores, these include efforts to:

- Manage/reduce disability claims (7.73)

- Improve employee job satisfaction (7.07)

- Impact business performance metrics and profitability (6.97)

- Improve employee daily health decisions at work (6.60)

- Attract or retain talented employees (6.26)

- Reduce the number of sick days (6.06)

- Reduce presenteeism (5.87), and

- Improve employee morale (5.66)

As in the previous example, readers who want to understand the relative importance of these items can divide the scores of any 2 items. For example, the 7.73 score for managing/reducing disability claims is 1.36 times as high and $36 \%$ higher than the 5.66 score for improving employee morale (ie, 7.73 / $5.66=1.36)$.

\section{Differences Among Employer Types}

The study also revealed differences among employers. For example, employers with mature health and wellness programs ( $\geq 6$ years old) had a broader view on how programs can deliver value. This may be attributed to their more sophisticated datagathering techniques, or perhaps because they have already demonstrated medical cost savings and want to report value in additional ways.

Jumbo-sized employers (those with 20,000 or more employees) placed more importance on health care cost reduction than did large employers (ie, their MaxDiff scores for this item were 14.81 and 12.73 , respectively). However, large employers (those with 3000-19,999 employees) placed more importance on morale, energy levels, and coworker relationships.

These findings suggest that the shift from ROI to VOI may be driven by the large employer segment that is seeing value in emerging metrics. Not surprisingly, companies with older workforces placed more importance on health care costs, sick days, and disability claims. Companies with younger workforces placed more importance on productivity.

Key Insight \#3. Many elements of program value are tracked. Employers reported that they track 6 or 7 metrics on average. Some of these metrics help them gauge the operational performance of their programs, while others help them understand program-related outcomes.

More than half of the employers surveyed tracked each of the following metrics to demonstrate health and wellness program value. Shown in order of most to least often tracked, these include:

- Health and wellness program engagement/participation (73\%)

- Health care costs $(70 \%)$

- Days absent (59\%)

- Wellness program satisfaction $(56 \%)$

- Health risk (54\%)

- Job satisfaction (53\%), and

- Safety $(52 \%)$.

It is interesting that 1 key metric-productivity-was missing from this list. Although improving productivity was one of the top 3 reasons for investing in health and wellness programs, only $34 \%$ of employers measure and track productivity. This may reflect uncertainties about how to measure it, especially if employers are differentiating between presenteeism, absenteeism, and other views about what productivity means.

Key insight \#4: Only a third of employers strongly agree they have the metrics they need to justify their investment in health and wellness programs.

Despite tracking multiple metrics to understand the value of health and wellness programs, the majority of employers do not believe they have the metrics they need to evaluate their programs. More than half of the employers reported that tracking safety, days absent, retention, and health care cost reduction are relatively easy. This is not surprising because these metrics can be evaluated within the human resources function.

The most difficult metrics to track, according to employers, were health risk, business performance/profitability, job satisfaction, food consumption, productivity, and employee morale. Fewer than $40 \%$ who tracked these metrics 
said it was easy to do so. These metrics are either more difficult to measure or require coordination across silos within the company.

\section{Limitations and the Opportunity Ahead}

Like any other survey, ours was not perfect. We focused on employers who had 5 or more health and wellness programs, so results may not be generalizable to those who had fewer. We could not find enough employers with fewer than 5 programs to allow any sort of generalization to them. Nevertheless, employers with more programs may see many more reasons to invest or to increase their investments in these programs.

Also, no survey panel construction and maintenance process can ever be perfect, so results may vary with other panels.

With these limitations in mind, the research revealed a core set of reasons that influence employer decisions to offer health and wellness programs. As employers start these programs, they may be focused on realizing health care cost savings. But as their programs evolve, they begin to prioritize other ways of valuing their investment.

This progression indicates that an employer does not have to show value in all areas at once; the value story can evolve over time. Although employers report being relatively confident in their ability to track health care costs and associated savings, they are less confident in their ability to track other emerging metrics. Working with researchers and consultants may help them see examples of detailed connections between emerging metrics and employee health and wellness program engagement.

A solid research or conceptually sound consulting process can help employers tell their VOI story by:

- Clearly crafting the reasons why they are offering health and wellness to their employees

- Identifying the metrics needed to support their health and wellness rationale

- Mapping out the source of each metric and how it can be accessed by using:

- Health plan data

- Vendor data

- In-house data

- Measuring the impact of health and wellness programs on their key metrics, and

- Aggregating findings into a meaningful dashboard based on their priorities.

In conclusion, we found that employers invest in health and wellness programs for many reasons, yet need some help measuring the full value of these programs. Doing so successfully will help avoid inappropriately casting the value of these programs solely in terms of their impact on health care expenditures. Research into the theoretical and empirical relationships between health and wellness investment strategies and outcomes will help guide work in this area as well.

\section{Author Disclosure Statement}

Drs. Ozminkowski and Serxner, and Ms. Marlo, Mr. Kichlu, Ms. Ratelis, and Ms. Van de Meulebroecke declared the following conflicts of interest with respect to the research, authorship, and/or publication of this article: The authors are employed by Optum, a UnitedHealth Group company, by TRC Market Research, a survey research firm, and by the National Business Group on Health. Some of the authors also own stock in their employer organizations. The opinions expressed in this commentary are those of the authors and may not represent the opinions of their employers. The authors retained full control of the content of this commentary.

The authors received the following financial support for this article: This work was funded by Optum.

\section{References}

1. Grossmeier J. Evaluating wellness programs: measuring the right things. Benefits Magazine. 2015;52(9):38-42.

2. Ozminkowski RJ. The development, implementation, and evaluation of corporate wellness programs. In: Burke RJ, Richardsen AM, eds. Corporate Wellness Programs: Linking Employee and Organizational Health. Cheltenham, UK: Edward Elgar; 2014:322.

3. Niebuhr S, Grossmeier J. Is it time for a broader approach? Recasting the value of "employee health" with a focus on workforce capability. Amer J Health Prom. 2015;29(6):TAHP6-TAHP9.

4. Smeltzer PA, Ozminkowski R, Musich S. Evaluating health promotion programs: applying best practices to evaluate and study the impact of health promotion program benefits, costs, health and productivity. March 8, 2011. http://www.corporate wellnessmagazine.com/worksite-wellness/impact-of-healthpromotion/. Accessed November 30, 2015.

5. Ozminkowski RJ. There's more to wellness program ROI than medical cost savings. June 9, 2014. http://www.hreonline.com/ HRE/view/story.jhtml?id=534357066. Accessed November 30, 2015.

6. Orme B. MaxDiff analysis: simple counting, individual-level logit, and HB. Sequim, WA: Sawtooth Software Inc.: 2009.

Address correspondence to:

Dr. Ronald J. Ozminkowski Consumer Solutions Group, Optum

315 East Eisenhower Parkway, Suite 305

Ann Arbor, Michigan 48108

E-mail: Ronald.ozminkowski@optum.com 